\title{
ESTILO DE VIDA DE PRATICANTES DE ATIVIDADES FÍSICAS EM ACADEMIAS DA TERCEIRA IDADE DE MARINGÁ - PR
}

João Victor Del Conti Esteves

Leonardo Vidal Andreato

Solange Marta Franzói de Moraes

Alessandra Regina Carnelozzi Prati

\section{Resumo}

$\mathrm{O}$ aumento da longevidade nas populações representa desafios importantes para a sociedade. $\mathrm{O}$ estilo de vida inadequado pode contribuir para que doenças se manifestem cada vez mais cedo, mas, por outro lado, o estilo de vida saudável pode ser o início da manutenção da saúde e prevenção de doenças. Acredita-se que atividade física regular, alimentação equilibrada e variada, boas relações sociais, assim como manutenção de comportamentos preventivos e controle de estresse sejam importantes para manter um estilo de vida saudável e equilibrado. Assim, esse trabalho teve como objetivo analisar o perfil do estilo de vida de praticantes de atividades físicas em Academias da Terceira Idade (ATI) da cidade de Maringá. Foram selecionados 45 indivíduos praticantes de atividades físicas na ATI de Maringá-PR. Como instrumento de medida foi utilizado o Pentáculo do Bem-estar, em que componentes como Nutrição (N), Atividade Física (AF), Comportamento Preventivo (CP), Relacionamento Social (RS) e Controle do estresse (CS) são parâmetros para diagnosticar o perfil do estilo de vida. A escala utilizada para análise dos dados indica como valores de: 0 a 1 = insuficiente (estilo de vida inadequado); 1 a $2=$ regular; 2 a 3 = adequado (estilo de vida saudável). Os resultados, em média, foram: $\mathrm{N}=1,80 ; \mathrm{AF}=2,06$; $\mathrm{CP}=2,48 ; \mathrm{RS}=2,05$ e $\mathrm{CS}=2,11$. Nota-se que o perfil do estilo de vida pode ser considerado moderado à positivo e, somente no componente nutrição o resultado foi considerado insatisfatório. Assim, espera-se que a atuação do profissional de Educação Física possa proporcionar consciência aos usuários, da necessidade de se ter hábitos saudáveis de vida.

\section{Palavras-Chave}

Estilo de vida; Idosos; Atividade física.

\section{LIFESTYLE OF PHYSICAL ACTIVITIES PRACTITIONERS IN THIRD AGE GYMS OF MARINGÁ - PR}

João Victor Del Conti Esteves

Leonardo Vidal Andreato

Solange Marta Franzói de Moraes

Alessandra Regina Carnelozzi Prati

\begin{abstract}
The increase in longevity in populations represents important challenges to society. The lifestyle inadequate can contribute to diseases to unfold increasingly early, but, on the other hand, the healthy lifestyle can be the beginning of health maintenance and disease prevention. It is believed that regular physical activity, balanced and varied diet, good social relations, as well as the preventive behaviors maintenance and stress management are important to maintain a healthy and balanced lifestyle. Thus, this study aimed to analyze the profile of lifestyle from physical activities practitioners in Third Age Gyms (TAG) of Maringá - PR City. It were selected 45 people engaged in physical activities in TAG from Maringá City. As measuring instrument it was used the Pentagram Welfare, components such as Nutrition (N), Physical Activity (PA), Preventive Behavior (PB), Social Relationship (SR) and Stress
\end{abstract}


Management (SM) are parameters to diagnose the lifestyle profile. The scale used for data analysis indicates as values from: 0 to $1=$ insufficient (inappropriate lifestyle); 1 to $2=$ regular; 2 to $3=$ adequate (healthy lifestyle). The results in average where: $\mathrm{N}=1,80 ; \mathrm{PA}=2,06 ; \mathrm{PB}=2,48 ; \mathrm{SR}=2,05$ and $\mathrm{SM}=$ 2,11 . It is noted that the lifestyle profile can be considered moderate to positive and, only in Nutrition component the result was considered unsatisfactory. Thus, it is expected that the performance of Physical Education professional can provide awareness to users about the need of having healthy living.

\section{Key-Words}

Lifestyle; Elderly; Physical activity. 


\section{INTRODUÇÃO}

A população mundial tem aumentado acentuadamente, em especial a população idosa. No Brasil, estimase que a população acima de 60 anos seja de 14 milhões de pessoas, sendo que projeções para 2025 é que serão mais de 32 milhões de idosos, correspondendo a 15\% da população do país (FORTI, CHACON MIKAHIL, 2004). Esse aumento deve-se a decorrência do aumento da expectativa média de vida associada à diminuição da taxa de fecundidade nos últimos tempos (AZEVEDO et al., 2005).

A expectativa de vida nas sociedades antigas era extremamente reduzida em relação à atual por problemas de saúde pública, doenças endêmicas e epidêmicas e baixo grau de educação das populações. A melhora nessas condições repercutiu não só no aumento da população idosa em todo o mundo, como também no envelhecimento com qualidade (AZEVEDO et al., 2005).

Existem muitas formas de entender o aumento da população idosa. Desde as teorias que citam fenômenos de modo isolado, bem como aquelas mais unificadoras. As melhorias das estruturas dos sistemas de saúde, aliadas aos incrementos das infra-estruturas de saneamento e habitação e às mudanças sociais nas áreas de educação, percepção e comportamento, ligados às áreas de saúde, tiveram papéis fundamentais na maior longevidade (MURRAY; CHEN, 1993).

De fato, as pessoas têm vivido mais e a preocupação com a saúde e uma vida com qualidade está cada dia maior. No entanto, uma vida com qualidade pode ser diferente de indivíduo para indivíduo.

Nahas (2001), define qualidade de vida como sendo a condição humana resultante de um conjunto de parâmetros individuais e sócio-ambientais, modificáveis ou não, que caracterizam as condições em que vive o ser humano. Considera-se como parâmetros individuais, moradia, meio de transporte, segurança, assistência médica, condições em que as pessoas trabalham, remuneração, educação, opções de lazer, meio ambiente, dentre outros. Já os parâmetros individuais são caracterizados como a hereditariedade e os componentes do estilo de vida: hábitos alimentares, controle do estresse, atividade física habitual, relacionamentos pessoais e comportamento preventivo.

Os parâmetros individuais são caracterizados como estilo de vida, que segundo autor acima, pode ser entendido como um conjunto de ações habituais que refletem as atitudes, os valores e as oportunidades na vida das pessoas.

$\mathrm{O}$ estilo de vida inadequado pode contribuir para que doenças se manifestem cada vez mais cedo, mas, por outro lado, o estilo de vida saudável pode ser o início da manutenção da saúde e prevenção de 
doenças, sejam elas infecto-contagiosas ou crônico-degenerativas. Dessa forma, acredita-se que atividade física regular, alimentação equilibrada e variada, boas relações sociais, assim como manutenção de comportamentos preventivos e controle de estresse sejam importantes para manter um estilo de vida saudável e equilibrado.

Quanto ao estilo de vida, parece que este passou a ser um dos mais importantes determinantes da saúde de indivíduos, grupos e comunidades (CHÓR, 1999) e ainda pode ser determinante na vida de idosos, que podem ter um envelhecimento com mais qualidade, não importando o quanto ainda vão viver, mas de que forma será o envelhecimento.

Assim, o objetivo do estudo foi analisar o perfil do estilo de vida de praticantes de atividades físicas em Academias da Terceira Idade (ATI) da cidade de Maringá.

\section{MATERIAS E MÉTODOS}

\section{Amostra}

O estudo caracteriza-se como descritivo-exploratório (THOMAS; NELSON, 2002), sendo a amostra foi composta por 45 indivíduos, sendo 12 homens e 33 mulheres, com idade média de 61,2 \pm 8 anos, praticantes de atividades físicas em Academias da Terceira Idade na cidade de Maringá.

\section{INSTRUMENTOS DE MEDIDA}

Como instrumento de medida utilizou-se o questionário Perfil de Estilo de Vida Individual, conhecido como Pentáculo do Bem-Estar (NAHAS; BARROS; FRANCALACCI, 2000), onde componentes como Nutrição, Atividade Física, Comportamento Preventivo, Relacionamento Social e Controle do estresse são parâmetros para diagnosticar o perfil do estilo de vida. Cada item contém três afirmações, numeradas de 1 a 3, nas quais o indivíduo manifestará sobre cada afirmação considerando a escala de 0 a 3 pontos, em que 0 significa "não fazer parte" do cotidiano, 1 significa "às vezes faz parte", 2 significa "quase sempre" e 3 significa "sempre faz parte" do cotidiano do indivíduo. Para análise do perfil do estilo de vida os valores obtidos foram empregados em tabelas e classificados como não satisfatório de 0 à 1 , regular de 1 à 2 e satisfatório de 2 à 3 .

\section{COLETA DE DADOS}

O questionário foi aplicado no ano de 2007 em diferentes ATIs da cidade. Este projeto foi submetido e aprovado pelo Comitê de Ética em Pesquisa da Pontifícia Universidade Católica do Paraná (parecer 464/2007-CEP/PUC-PR). Todos os indivíduos que concordaram em participar do estudo assinaram o 
$\overline{\text { Termo de Consentimento Livre e Esclarecido, estando cientes que a qualquer momento poderiam }}$ questionar os procedimentos ou desistir da pesquisa.

\section{ANÁLISE ESTATÍSTICA}

Para o tratamento dos dados foi utilizada a estatística descritiva (média \pm desvio padrão) para caracterização da amostra. Foi realizado teste t-student para as comparações entre gêneros, adotando-se o valor de $\mathrm{p}<0,05$ para apontar as diferenças significativas. As análises foram feitas utilizando-se o programa GRAPHPAD PRISM (versão 4.3).

\section{RESULTADOS E DISCUSSÕES}

Os resultados do perfil de estilo de vida dos praticantes de atividades físicas das Academias da Terceira Idade de Maringá são mostrados na tabela 1 discriminando os diferentes componentes do estilo de vida.

Tabela 1- Comparação entre gêneros de perfil de estilo de vida de idosos praticantes de atividades físicas em ATIs de Maringá.

\begin{tabular}{lll}
\hline Componentes & Homens $(\mathbf{n}=\mathbf{1 2})$ & Mulheres $(\mathbf{n}=\mathbf{3 3})$ \\
\hline $\mathbf{N}$ & $2,03 \pm 0,1$ & $1,91 \pm 0,21$ \\
AF & $2,36 \pm 0,46$ & $2,17 \pm 0,51$ \\
$\mathbf{C P}$ & $2,56 \pm 0,39$ & $2,73 \pm 0,22$ \\
$\mathbf{R S}$ & $2,33 \pm 0,46$ & $2,16 \pm 0,58$ \\
CS & $2,39 \pm 0,59$ & $2,25 \pm 0,44$ \\
Idade & $66,83 \pm 7,55^{*}$ & $59,21 \pm 7,2$ \\
\hline
\end{tabular}

$\mathbf{n}=$ número de indivíduos da amostra; $\mathbf{N}=$ Nutrição; $\mathbf{A F}=$ Atividade Física; $\mathbf{C P}=$ Comportamento Preventivo; RS= Relacionamento Social; $\mathbf{C S}=$ Controle de Estresse. Os valores são apresentados em média e desvio padrão. ${ }^{*}=\mathrm{p}<0,001$.

Ambos os grupos não apresentaram diferenças estatisticamente significativas $(p>0,05)$ no perfil relacionado à nutrição, atividade física, comportamento preventivo, relacionamento social e controle de estresse. No entanto, o grupo masculino apresentou idade muito superior ao grupo feminino $(p<0,001)$.

Embora, mesmo não tendo sido constatadas diferenças significativas entre os grupos, no componente nutrição os homens apresentaram melhores resultados, com níveis satisfatórios no componente $(2,03 \pm$ $0,1)$. Em contrapartida, nesse componente as mulheres são consideradas com níveis regulares. Observa-se 
$\overline{\text { que nos outros componentes não houve diferença de classificação, mostrando uma certa uniformidade }}$ entre os praticantes de atividades físicas nas ATIs.

A figura 1 apresenta resultados dos componentes: Nutrição (N), Atividade Física (AF), Comportamento Preventivo (CP), Relacionamento Social (RS) e Controle de Estresse (CS).

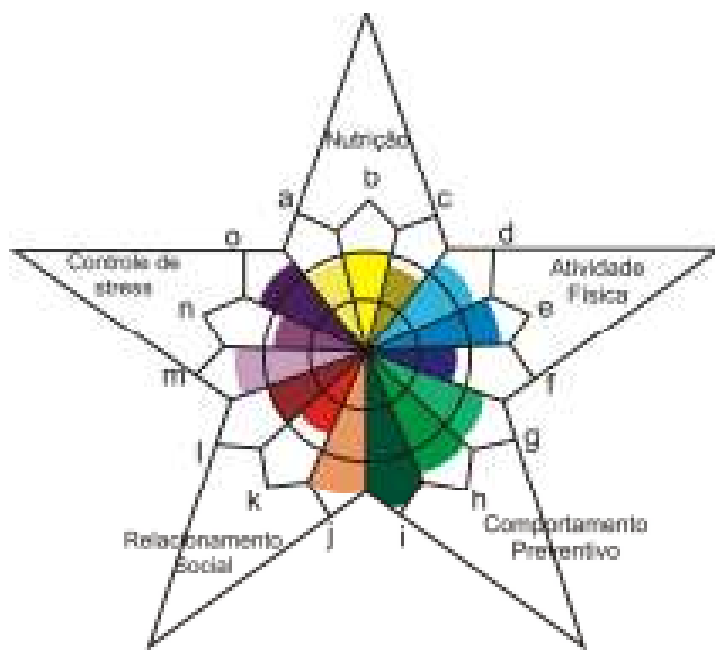

$$
\begin{array}{r}
\mathrm{N}=1,94 \\
\mathrm{AF}=2,22 \\
\mathrm{CP}=2,68 \\
\mathrm{RS}=2,21 \\
\mathrm{CS}=2,29
\end{array}
$$

Figura 1- Análise do Perfil do Estilo de Vida dos idosos praticantes de atividades físicas nas Academias de Terceira Idade de Maringá.

Analisando a Figura 1 observa-se que no componente Nutrição as pessoas analisadas apresentam resultado regular $(1,94 \pm 0,16)$. Neste sentido, sabe-se que com o envelhecimento, mudanças antropométricas, neuromusculares e metabólicas acometem os idosos (MATSUDO et al., 2000; JANSSEN, 2009). Em relação à composição corporal, ocorre uma perda acentuada de massa muscular (FRONTERA et al., 2000; JANSSEN et al., 2000) e uma redistribuição de gordura da periferia para a região abdominal, contribuindo para um aumento da gordura corporal (ZAMBONI et al. 1997; CAMPOS et al., 2000). Sendo assim, uma alimentação saudável, diversificada, rica em nutrientes essenciais torna-se de suma importância nessa fase da vida, seja para prevenção de doenças ou para manutenção da saúde.

O componente atividade física pode ser considerado satisfatório $(2,22 \pm 0,42)$. Neste âmbito, são vários os estudos que relacionam a atividade física e o exercício físico com a população idosa, principalmente apontando suas modificações e melhoras. O exercício físico é capaz de promover melhora no equilíbrio, na coordenação e agilidade de idosos (SILVA et al., 2008), gerar reduções significativas na Pressão Arterial e Freqüência Cardíaca de Repouso (KRINSKI et al., 2006), prevenir e reduzir os sintomas depressivos (MORAES et al., 2007), melhorar a saúde mental (COELHO et al., 2009) e diminuir o risco de demência (BENEDETTI et al., 2008), dentre muitos outros benefícios. 
No entanto, percebe-se, que mesmo entre os praticantes de atividade física nas ATIs, muitos deles não têm uma prática regular no seu dia-a-dia o qual associado a uma alimentação inadequada pode trazer conseqüências para a saúde como o aumento da gordura corporal. Tal aumento da adiposidade também está associado a doenças crônicas não transmissíveis, tais como: diabetes tipo 2, doenças cardiovasculares, dislipidemia, alguns tipos de câncer e está diretamente ligada ao aumento da taxa de mortalidade (FIATARONE- SINGH, 1998; DISHMAN et al., 2006; LAU et al., 2007). Deste modo, a prevalência de sobrepeso e obesidade crescente entre os indivíduos acima de 60 anos produz conseqüências adversas para a saúde, aumentando a incidência de várias moléstias (ORTEGA et al., 1995; KRAEMER et al., 2009).

O componente comportamento preventivo por sua vez apresenta o melhor resultado entre os participantes da pesquisa, sendo considerado satisfatório $(2,68 \pm 0,27)$. Constata-se neste componente a maior preocupação e o maior comprometimento dos idosos. Tal resultado corrobora com estudo realizado por Miranda et al. (2007) com uma amostra de idosos, no qual foi observado que dentre os componentes do estilo de vida, o item que mais se destacou foi o comportamento preventivo.

Sobre este aspecto, em nosso estudo, a maioria dos idosos consultava o médico regularmente, não ingeriam mais de uma dose de bebida alcoólica por dia e nem fumavam, procuravam seguir as normas de trânsito, sempre usavam o cinto de segurança e quando dirigiam nunca ingeriam álcool.

O relacionamento social entre os praticantes de atividades físicas das ATIs foi considerado também satisfatório $(2,21 \pm 0,55)$. Percebe-se que mesmo entre os participantes da pesquisa, que muitas vezes relatam sobre a presença nas academias para conversar com outras pessoas, onde para muitos a academia é o único lugar para que eles se interajam e sintam-se importantes entre as pessoas, os relacionamentos não são tão efetivos. Muitos dos idosos que frequentam a academia, se sentem sozinhos em casa, com poucos amigos e a presença da família cada vez mais reduzida.

Em relação ao componente estresse o resultado foi considerado satisfatório $(2,29 \pm 0,48)$. O estresse pode afetar a saúde mental de crianças, adolescentes, adultos e idosos, pessoas de todas as raças, de ambos os sexos, níveis educacionais e de rendimento. No entanto, estudos indicam uma prevalência maior de estresse entre adultos e idosos (MARINHO, 2002).

O controle do estresse pode ser determinante no desenvolvimento de doenças e uma qualidade de vida comprometida e está associado a diversas doenças, entre elas destacam-se as doenças cardíacas, hipertensão, insônia, enxaqueca, desordens alimentares entre outras. (LIPP, 1994). 
Nahas (2001) afirma que nunca é demais lembrar que a Educação Física, assim como outras profissões da saúde, tem um importante papel social no processo educativo para um estilo de vida saudável e para uma vida com mais qualidade. E talvez se profissionais da área tivessem uma maior preocupação de intervenção na vida das pessoas idosas, esses poderiam ter um envelhecimento com mais qualidade.

\section{CONCLUSÃO}

Pode-se concluir de maneira geral que o perfil do estilo de vida dos idosos praticantes de atividade física nas Academias da Terceira Idade de Maringá pode ser considerado satisfatório, no entanto, somente no componente nutrição o resultado foi considerado regular.

Assim, espera-se que a atuação do profissional de Educação Física possa proporcionar consciência aos idosos, da importância da se manter um de um estilo de vida saudável, e quando necessário intervir positivamente nos parâmetros que compõe a qualidade de vida.

\section{REFERÊNCIAS}

AZEVEDO, L. F.; A., D. O.; OKUMA, S. S. Envelhecimento e Exercício Físico. In: NEGRÃO, C. E.; BARRETTO, A. C. P.(Ed.). Cardiologia do Exercício: Do Atleta ao Cardiopata. Barueri - SP: Manole, 2005.

BENEDETTI, T. R. B.; et al. Atividade fisica e estado de saúde mental de idosos. Revista de Saúde Pública, v. 42, n. 2, p.302-307, 2008.

CAMPOS, M. T. F. S.; MONTEIRO, J. B. R.; ORNELAS, A. P. R. C. Fatores que Afetam o Consumo Alimentar e a Nutrição do Idoso. Revista de Nutrição, Campinas; v. 13, n. 3, p. 157-165, 2000.

CHÓR, D. Saúde pública e mudanças de comportamento: uma questão contemporânea. Caderno de Saúde Pública v. 15, n. 2, p. 423-425, 1999.

COELHO, F. G. M. et al., Atividade física sistematizada e desempenho cognitivo em idosos com demência de Alzheimer: uma revisão sistemática. Revista Brasileira de Psiquiatria, v. 31, n. 2, p.163-70, 2009.

DISHMAN, R. K. et al.; Neurobiology of exercise. OBESITY, v. 14, n. 3, p. 345-356, 2006. 
FIATARONE- SINGH, M. A. Body composition and weight control in older adults. In: LAMB, D. R.; MURRAY, R. (Eds). Perspectives in exercise science and sports medicine: exercise, nutrition and weight control. Carmel:Cooper; v.11, p.243-288, 1998.

FORTI, V. A. M.; MIKAHIL, M. P. T. C. Qualidade de vida e atividade fisica na terceira idade. In: GONÇALVES, A.; VILARTA, R (Org). Qualidade de vida e atividade física: explorando teorias e práticas. Barueri - SP: Manole, P.227-249, 2004.

FRONTERA, W. R. et al., Aging of skeletal muscle: a 12-yr longitudinal study. J Appl Physiol, v. 88, n. 4, p. 1321-1326, 2000.

JANSSEN, I.; et al. Skeletal muscle mass and distribution in 468 men and women aged $18 e 88 \mathrm{yr}$. J Appl Physiol, v. 89, n. 1, p. 81-88, 2000.

JANSSEN, I. Influence of age on the relation between waist circumference and cardiometabolic risk markers. Nutrition, Metabolism \& Cardiovascular Diseases, v. 19, p. 163-169, 2009.

KRAEMER, W. J., et al., T. American College of Sports Medicine. Position Stand. Exercise and Physical Activity for Older Adults. Med Sci Sports Exerc, v. 41, n. 7, p. 1510-1530, 2009.

KRINSKI, K.; et al., Efeito do exercício aeróbico e resistido no perfil antrométrico e respostas cardiovasculares de idosos portadores de hipertensão. Acta Scientiarum Health Science, v. 28, n.1, p.7175, 2006.

LAU, D. C. W. et al., 2006 Canadian clinical practice guidelines on the managemente and prevention of obesity in adults and children [summary]. CMAJ, v. 176, n. 8, p. 1-13, 2007.

LIPP, M. N.; ROCHA, J. C. Estresse, hipertensão arterial e qualidade de vida. Campinas, SP: Papirus, 1994.

MARINHO, C. S. Estilo de Vida e Indicadores de Saúde de Estudantes Universitários da UNIPLAC. Tese - (Mestrado) Faculdade de Educação Física, Universidade Federal de Santa Catarina, Florianópolis, 2002.

MATSUDO, S. M.; MATSUDO, V. K. R.; NETO, T. L. B. Impacto do envelhecimento nas variáveis antropométricas, neuromotoras e metabólicas da aptidão física. Revista Brasileira de Ciência e Movimento, v. 8, n. 4, p.21-32, 2000.

MIRANDA, L. M.; GUIMARÃES, A. C. A.; SIMAS, J. P. N. Estilo de vida e hábitos de lazer de freiras e padres idosos de Florianópolis -SC. Revista Brasileira de Ciência e Movimento, v. 15, n. 1, p. 15-22, 2007.

MORAES, H. et al., O exercício físico no tratamento da depressão em idosos: revisão sistemática. Revista de Psiquiatria do Rio Grande do Sul, v. 29, n. 1, p. 70-79, 2007. 
MURRAY, C. J. L.; CHEN, L. C. Search of contemporany theory for understanding mortality change. Soc Sci Méd, 6:143-155, 1993.

NAHAS, M. V. Atividade física, saúde e qualidade de vida: conceitos e sugestões para um estilo de vida ativo. Londrina: Midiograf, 2001.

NAHAS, M. V.; BARROS, M. V. G.; FRANCALACCI, V. L. O pentáculo do bem-estar: base conceitual para a avaliação de estilo de vida de indivíduos ou grupos. Revista Brasileira de Atividade Física e Saúde, 5(2), 48-89, 2000.

ORTEGA, R. M. et al., Eating behavior and energy and nutrient intake in overweight/obese and normalweight Spanish elderly. Ann. Nutr. Metab, v. 39, p. 371-378, 1995.

SILVA, A. et al. Equilíbrio, Coordenação e Agilidade de Idosos Submetidos à Prática de Exercícios Físicos Resistidos. Revista Brasileira de Medicina do Esporte, v. 14, n. 2, p. 88-93, 2008.

THOMAS, J. R.; NELSON, J. K. Métodos de pesquisa em atividade fisica. São Paulo: Artmed, 2002. ZAMBONI, M. et al. Effects of age on body fat distribution and cardiovascular risk factors in women. Am J Clin Nutr, v. 66, n. 1, p. 111-115, 1997. 


\section{João Victor Del Conti Esteves}

Universidade Estadual de Maringá (UEM)

e-mail: joaovesteves@gmail.com

Especialização em Prescrição Personalizada de Exercícios Físicos

Personal Training (UEM)

aluno do programa de mestrado em Educação Física UEM/UEL.

\section{Leonardo Vidal Andreato}

Universidade Federal de São Paulo (UNIFESP)

e-mail:vidal.leo@hotmail.com

\section{Solange Marta Franzói de Moraes}

Universidade Estadual de Maringá (UEM)

e-mail: smfmoraes@uem.br

\section{Alessandra Regina Carnelozzi Prati \\ Universidade Estadual de Maringá (UEM) \\ e-mail lecarnelozzi@hotmail.com}

\section{Referência do Artigo}

\section{ABNT}

ESTEVES, J. V. D. C.; ANDREATO, L. V.; MORAES, S. M. F. PRATI, A. R. C. Estilo de vida de praticantes de atividades físicas em academias da terceira idade de Maringá - PR. Conexões, v. 8, n. 1, p. 119-129, 2010

\section{APA}

Esteves, J. V. D. C.; Andreato, L. V.; Moraes, S. M. F. \& Prati, A. R. C.(2010). Estilo de vida de praticantes de atividades físicas em academias da terceira idade de Maringá - PR. Conexões, 8(1), 119-129.

\section{VANCOUVER}

Esteves, JVDC, Andreato, LV, Moraes, SMF. Prati, ARC. Estilo de vida de praticantes de atividades físicas em academias da terceira idade de Maringá - PR. Conexões, 2010; 8(1): 119-129. 